\title{
The public health benefits of reducing greenhouse gas emissions
}

\section{Andrew Haines}

Public Health and Primary Care,

London School of Hygiene and Tropical Medicine, UK

Abstract of the keynote address at the 2010 Fenner Conference, Australian Academy of Science, Canberra, 22-23 June 2010 (reproduced with permission).

Climate change will harm human health, and successful strategies to reduce greenhouse gas emissions will restrict that harm. But studies published in The Lancet ${ }^{1}$ late last year showed that appropriate mitigation strategies will themselves have additional and independent effects on health, most of them beneficial. The potential value of these co-benefits has not so far been given sufficient prominence in international negotiations.

These studies, supported by a global partnership of funders, were undertaken by an international multidisciplinary group of researchers with the aim of informing discussions at the 2009 Copenhagen conference of parties to the UN Framework Convention on Climate Change. Each focused on one sector which is a major source of greenhouse-gas emissions. These sectors are: household energy use, urban land transport, electricity generation, and food and agriculture. A fifth study reviewed the effect on health of short-lived greenhouse pollutants, which are produced in several sectors.

Each of the sectoral studies examined the health implications of actions in both high-income and low-income countries designed to reduce the release of carbon dioxide $\left(\mathrm{CO}_{2}\right)$ and other greenhouse gases through a number of case studies. In line with the recommendations of the UK Committee on Climate Change, each would yield reductions by 2030 that are broadly consistent with the aim of meeting a global 50\% reduction target (compared with 1990) by 2050 , and an $80 \%$ reduction in emissions for high-income countries. The studies demonstrate the potential improvements in health through a range of mechanisms such as increasing active transport (walking and cycling) in cities, reducing exposure to indoor and outdoor air pollution and reducing consumption of animal source saturated fats. These co-benefits can offset, at least in part, the costs associated with implementing strategies to reduce greenhouse gas emissions. Future research should be directed at exploring the potential cobenefits in a range of settings, reducing uncertainties and assessing the health effects of other strategies, such as biofuels or carbon capture and storage, which were not covered by this program of research. It is clear however that a lower carbon and more sustainable economy could result in substantial improvements in public health.

\section{Reference}

1. Haines A, McMichael AJ, Smith KR, Roberts I, Woodcock J, Markandya A et al. Public health benefits of strategies to reduce greenhouse-gas emissions: overview and implications for policy makers. Lancet 2009; 374: 2104-14. doi:10.1016/ S0140-6736(09)61759-1 